International Journal of Modern Physics A

(C) World Scientific Publishing Company

\title{
PRODUCTION OF $\eta$ MESONS IN PROTON-PROTON COLLISIONS CLOSE TO THRESHOLD
}

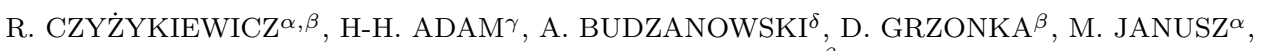

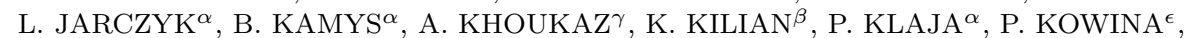
P. MOSKAL ${ }^{\alpha, \beta}$, W. OELERT ${ }^{\beta}$, C. PISKOR-IGNATOWICZ ${ }^{\alpha}$, J. PRZERWA ${ }^{\alpha}$, T. ROŻEK ${ }^{\beta, \zeta}$,

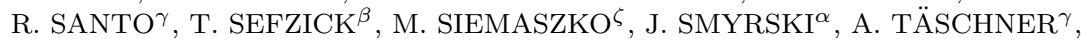

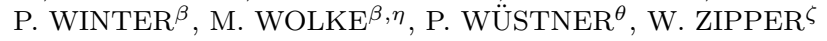
(COSY-11 Collaboration)

${ }^{\alpha}$ Institute of Physics, Jagellonian University, Cracow, PL-30-059, Poland

${ }^{\beta} I K P$, Forschungszentrum Jülich, Jülich, D-52425, Germany

${ }^{\gamma}$ IKP, Westfälische Wilhelms-Universität, Münster, D-48149, Germany

${ }^{\delta}$ Institute of Nuclear Physics, Cracow, Poland

${ }^{\epsilon}$ Accelerator Division, Gesellschaft für Schwerionenforschung, Darmstadt, Germany $\zeta$ Institute of Physics, University of Silesia, Katowice, Poland ${ }^{\eta}$ Svedberg Laboratory, Uppsala, Sweden

${ }^{\theta}$ ZEL, Forschungszentrum Jülich, Jülich, Germany

\begin{abstract}
A brief experimental overview on the close-to-threshold $\eta$ meson production in protonproton interactions is presented and the available observables in measurements with unpolarized and polarized beam and target are discussed.
\end{abstract}

Keywords: eta, close-to-threshold meson production, eta-N interaction, analysing power.

\section{Measured observables}

The still not well established production mechanism of the $\eta$ meson as well as its interaction with protons can be investigated via measurements of the following observables available in proton-proton scattering:

\subsection{Total cross section}

Measurements of the total cross section for the $p p \rightarrow p p \eta$ reaction have been performed at various excess energies in many laboratories worldwide 1213415. The existing data, gathered utilizing different experimental techniques are in excellent agreement with each other ${ }^{a}$. Theoretical considerations of the excitation function led in most cases to the conclusion that close-to-threshold production of the $\eta$ mesons in proton-proton collisions proceeds predominantly via the excitation and deexcitation of the negative parity $\mathrm{S}_{11}(1535)$ resonance ${ }^{\mathrm{b}}$. Despite the very good

${ }^{\text {a }}$ Due to limited space we are not presenting here the figure of the excitation function for the $p p \rightarrow p p \eta$ reaction. For an overview picture see for instance Fig. $8 \mathrm{a}$ in 6

${ }^{b}$ For an overview of theoretical models see section 7.3 of 10 and references therein. 
agreement between different theoretical models as far as the leading $\eta$ creation mechanism is concerned, there are a lot of discrepancies when trying to explain which out of the considered exchanged mesons plays the dominant role in the excitation of the $\mathrm{S}_{11}$. As it was shown by Nakayama et al. $\underline{8}$, both pseudoscalar and vector meson exchanges result in equally well descriptions of the excitation function for the $p p \rightarrow p p \eta$ reaction close to threshold.

\subsection{Angular distributions}

Measurements of the close-to-threshold angular distributions of the $\eta$ meson emission in the centre-of-mass system at excess energies of $\mathrm{Q}=15 \stackrel{[3}{3}, 15.5^{4}, 37^{2}$, and $41 \mathrm{MeV}[3$ showed rather flat distributions. It is concluded from these data that in the lower excess energy range the $\eta$ meson is mainly produced in the $s$-wave, while at higher excess energies the influence of the $p$-wave starts to play an important role 9 . The contribution from the $d$-wave seems to be negligible. Also the proton's angular distributions of the centre-of-mass emission angle $\frac{344}{4}$ show an isotropic behaviour. At $\mathrm{Q}=15 \mathrm{MeV}$ the data indicate dominance of the ${ }^{3} P_{0} \rightarrow{ }^{1} S_{0} s$ transition distorted slightly by the contribution from the ${ }^{1} S_{0} \rightarrow{ }^{3} P_{0} s$. At $\mathrm{Q}=40 \mathrm{MeV}$ there is visible an additional presence of the ${ }^{1} D_{2} \rightarrow{ }^{3} P_{2} s$ transition 9 . Contributions from the other partial waves were found to be of minor importance. In contradiction to these results, the emission plane of the $p p \rightarrow p p \eta$ reaction was found to be anisotropic $4 \mathrm{c}$. This effect has not been explained theoretically yet.

\subsection{Invariant mass distributions}

The high statistics production of $\eta$ mesons in proton-proton collisions at $Q=$ 15.5 $\mathrm{MeV}$ allowed to perform a Dalitz plot analysis of the 3-body final state 4 . Surprisingly, beside the clear enhancement seen in the lower range of the invariant mass distribution of the $p p$ subsystem, originating in the strong proton-proton final state interaction, the presence of a wide bump in the upper range of this plot has been observed. Different approaches have been applied in order to explain the origin of this wide bump. Nakayama et. al. 9 described the distribution by introducing $P$-waves in the $p p$ subsystem. This approach, although in agreement with the shapes of both $p p$ and $p \eta$ invariant masses, fails when explaining the shape of the excitation function for the $p p \rightarrow p p \eta$ reaction in the range below $\mathrm{Q}=40 \mathrm{MeV}$. It is also worth to mention that an indication of such a bump was also seen in the $p p$ invariant mass distribution at $\mathrm{Q}=4.5 \mathrm{MeV} 4$, where one expects the presence of $S$-waves only. A three-body calculation performed by Fix and Arenhövel 12 could also describe the bump in the $p p$ invariant mass at $\mathrm{Q}=15.5 \mathrm{MeV}$ well, however it fails in explaning the origin of the peak in the low range of $p p$ invariant mass. It also doesn't reproduce the shape of the $p p$ invariant mass at $\mathrm{Q}=41 \mathrm{MeV}$. The $p \eta$ invariant mass distributions were not described in this approach. Solution based on the

${ }^{\mathrm{c}}$ Which is in line with the result by 11 
parametrization of the reaction amplitude proposed by Deloff 13 resulted in a good description of the $p p$ and $p \eta$ invariant masses at $\mathrm{Q}=15.5 \mathrm{MeV}$. However, it fails at $\mathrm{Q}=40 \mathrm{MeV}$. Another model proposed by the same author, based on a three-particle pair-wise approach via the hyperspherical harmonics 14 , led to a good description of $p p$ and $p \eta$ invariant mass distributions at $\mathrm{Q}=15.5 \mathrm{MeV}$. Solutions at $\mathrm{Q}=40 \mathrm{MeV}$ as well as the excitation function were not discussed in frame of this model.

\subsection{Analysing power}

Polarisation observables should provide a more detailed information on the dynamics of the $\eta$ meson creation in hadronic collisions, due to their sensitivity to the interference terms between $P s$ and $P p$ waves. The first attempt to measure the proton analysing power for the $\overrightarrow{p p} \rightarrow p p \eta$ has been undertaken by the COSY-11 group, with a set of data at $\mathrm{Q}=40 \mathrm{MeV}$ 15]. The data, within their rather large error bars, are consistent with zero, which indicates the absence of higher-than- $s$ partial waves in $\eta$ production at $\mathrm{Q}=40 \mathrm{MeV}$. The $P s$ and $P p$ interference term as well as the sum of the $(P p)^{2}$ and $S s S d$ interference terms were extracted in the analysis and are equal to $(0.003 \pm 0.004) \mu \mathrm{b}$ and $(-0.005 \pm 0.005) \mu \mathrm{b}$, respectively. In particular, this may indicate that there is no interference between the $P s$ and $P p$ waves. Recently, the DISTO collaboration has obtained the set of $\mathrm{A}_{y}$ data at excess energies far from threshold, which surprisingly also turned out to be consistent with zero within the error bars 5 .

\section{Prospectives}

Beside the upcoming data from the COSY-11 collaboration for the proton analysing power in the $\overrightarrow{p p} \rightarrow p p \eta$ reaction at $\mathrm{Q}=10$ and $37 \mathrm{MeV}^{\mathrm{d}}$ it was proposed to measure the spin correlation function ${ }^{\mathrm{e}}$ for the $\vec{p} \vec{p} \rightarrow p p \eta$ reaction at COSY 18. The latter would be possible at an external target station at COSY with a frozen spin target. For a high efficiencies in the $p p \eta$ event separation the WASA detector 19, which will be moved to COSY in the near future, would be at best suited. The spin correlation function is an model-independent direct measure of the contribution of the ${ }^{3} P_{0} \rightarrow{ }^{1} S_{0} s$ transition in the creation of the $\eta$ meson 910. Performing this experiment in the range of the excess energies, where the only contributions to the production amplitude are those from the ${ }^{3} P_{0} \rightarrow{ }^{1} S_{0} s$ and ${ }^{1} S_{0} \rightarrow{ }^{3} P_{0} s$ transitions, one will be able to exctract contributions from the both transitions in this excess energy range.

${ }^{\mathrm{d}}$ Status of the analysis will be reported in 16 .

e Theoretical predictions for the $\mathrm{C}_{x x}$ values are given for instance in 917 . 


\section{Acknowledgments}

The work has been supported by the European Community - Access to Research Infrastructure action of the Improving Human Potential Programme, by the DAAD Exchange Programme (PPP-Polen), by the Polish State Committe for Scientific Research (grants No. 2P03B07123 and PB1060/P03/2004/26), and by the Research Centre Jülich.

\section{References}

1. E. Chiavassa et al., Phys. Lett. B322, 270 (1994); H. Calén et al., Phys. Lett. B366, 39 (1996); H. Calén et al., Phys. Rev. Lett. 79, 2642 (1997); F. Hibou et al., Phys. Lett. B438, 41 (1998); H. Calén et al., Phys. Rev. C 58, 2667 (1998); J. Smyrski et al., Phys. Lett. B474, 180 (2000).

2. H. Calén et al., Phys. Lett. B458 190 (1999).

3. M. Abdel-Bary et al., Eur. Phys. J. A16 127 (2003).

4. P. Moskal et al., Phys. Rev. C 69, 025203 (2004).

5. F. Balestra et al., Phys. Rev. C 69, 064003 (2004).

6. P. Moskal, M. Wolke, A. Khoukaz and W. Oelert Prog. Part. Nucl. Phys. 49, 1 (2002).

7. C. Caso et al., Eur. Phys. J. C3 1 (1998).

8. K. Nakayama et al., Phys. Rev. C 65045210 (2002).

9. K. Nakayama et al., Phys. Rev. C 68, 045201 (2003).

10. C. Hanhart et al., Phys. Rep. 397155 (2004).

11. E. Roderburg et al., Acta Phys. Pol. B31 2299 (2000).

12. A. Fix and H. Arenhövel, Phys. Rev. C 69, 014001 (2004).

13. A. Deloff, Phys. Rev. C 69, 035206 (2004).

14. A. Deloff, nucl-th/0406069

15. P. Winter et al., Phys. Lett. B544, 251 (2002); Erratum-ibid B553, 339 (2003).

16. R. Czyżykiewicz et al., Contribution to the COSY-11 Extended Meeting (to be published in Schriften des FZ-Jülich, 2004).

17. M.P. Rekalo et al., Phys. Rev. C 55, 2630 (1997).

18. P. Winter, R. Czyżykiewicz et al., nucl-ex/0406034

19. J. Zabierowski et al., Phys. Scripta T99, 159 (2002). 